\title{
A review: drinking water and wastewater quality assessment in ruminant farms
}

\begin{abstract}
Water is an essential part in any livestock production and it is more so in ruminant production that is highly water intensive. Water is not only essential as a medium of animal metabolism, but it is also an important cleaning medium. Therefore, it is important that the quality of drinking water is maintained at a certain level so as to lessen the possible adverse effects on the animals. Despite there has being some standards set by the various agencies on the minimal water quality deemed suitable for livestock consumption, they are still vague and this, combined with poor compliance and the water sourcing of dubious sources in ruminant farms makes it hard to regulate the drinking water quality in ruminant farms. This, in turn, might lead to possible adverse effects in livestock production due to water contaminants. At the same time, most ruminant farms in Malaysia do not do proper treatment of their wastewater before releasing them into water bodies such as drains, rivers and lakes which could lead to possible adverse effects to the environment.
\end{abstract}

Keyword: Drinking water; Wastewater; Quality; Livestock 